\title{
Toward Singlet Oxygen Delivery at a Measured Rate: A Self- Reporting Photosensitizer
}

\author{
Sundus Erbas-Cakmak ${ }^{\dagger}$ and Engin U. Akkaya* ${ }^{\dagger}, \ddagger$ \\ ${ }^{\dagger}$ UNAM-National Nanotechnology Research Center and Department of Chemistry, Bilkent University, Ankara TR-06800, Turkey
}

Supporting Information

ABSTRACT: A Bodipy-based energy transfer cassette with a singlet oxygen reactive linker between the donor and acceptor modules has an interesting emergent property, if the acceptor module is also a photosensitizer. Singlet oxygen produced by the photosensitizer reacts rapidly with the molecule itself to liberate the energy donor, resulting in an enhanced fluorescence emission. The result is a self-reporting photosensitizer providing an assessment of the singlet oxygen production rate under the operational conditions.

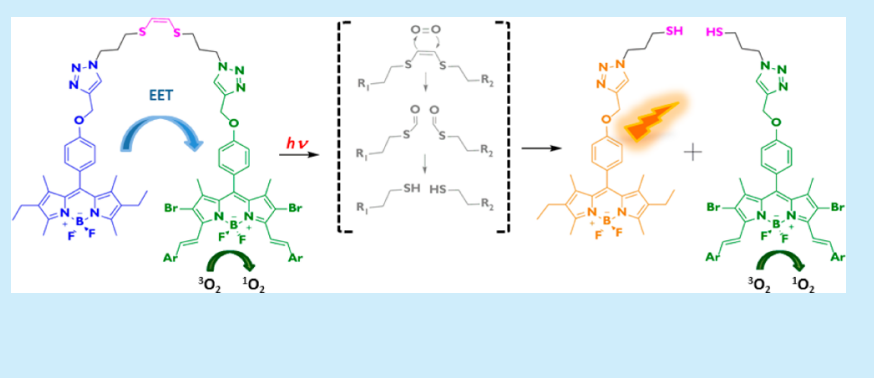

S ensing analytes in solution is highly important both for the elucidation of the processes they are involved in and/or for determining therapeutic/diagnostic approaches in diseases where corresponding analytes are markers for various biological anomalies. ${ }^{1,2}$ Apart from using analyte responsive molecules as reporters, activatable therapeutic systems have been developed as well, where the disease related parameter is used to initiate a chemical/physical transformation in the therapeutic agent to alter its activity. Activatable photosensitizers are one of such therapeutics with a modulated activatability property. ${ }^{3-8}$ Exchange of ideas between the fields of therapeutic design and molecular sensors is expected to yield great improvements in personalized treatment and diagnostics. However, one of the main problems not addressed so far in this overlap area, is the inadequacy of measuring the effect of any therapeutics directly. Rather, the biological effect is analyzed on a cellular level. For the photosensitizers, the effect of light, oxygen concentration, cell penetration, and dark-toxicity determines the overall effect of the photosensitizer on the cell; ${ }^{9,10}$ hence, one has to be cautious in speaking about the efficiency of singlet oxygen production of a photosensitizer in a cell-culture experiment since the observed outcome is a cumulative result, namely cell death.

In this work, we address the issue of direct monitoring of photosensitizer activity with the use of a singlet oxygen $\left({ }^{1} \mathrm{O}_{2}\right)$ labile linker between the fluorophore and a photosensitizer, where the former is an electronic energy transfer (EET) donor and the latter being an EET acceptor (Figure 1, BOD 2). The rationale behind the design is such that EET from the fluorophore (FL, blue module in Figure 1) to the photosensitizer (PS, green module on BOD 2) quenches the emission of the FL to a great extent. PS and FL are attached to one another with $(Z)$ - 1,2-bis(alkylthio)ethene bridge due to the fact that ${ }^{1} \mathrm{O}_{2}$ susceptibility of this electron-rich olefinic linker is extensively exploited by us and others. ${ }^{11-16}$

According to the design, ${ }^{1} \mathrm{O}_{2}$ generated by the PS upon irradiation with red light is to act on the molecule itself and

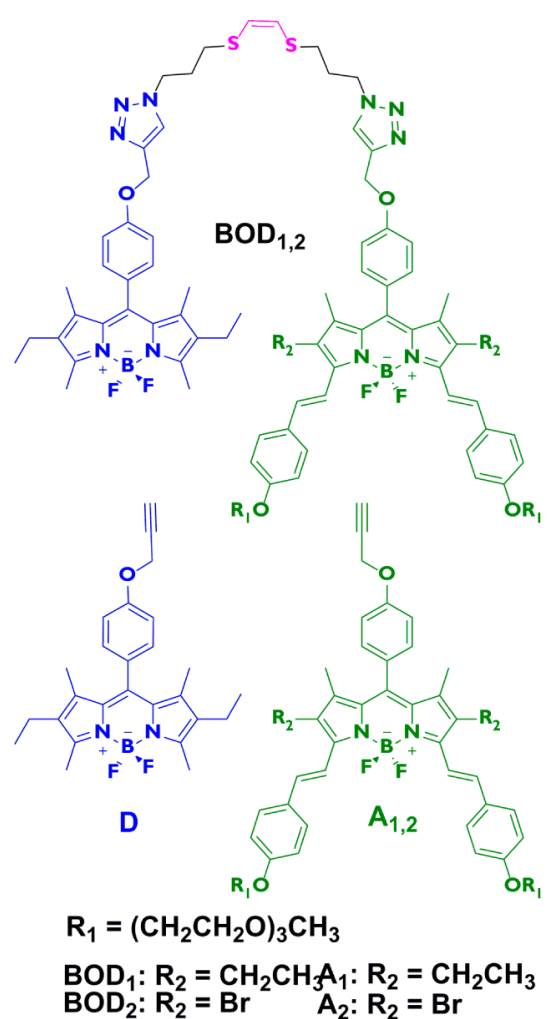

Figure 1. Structure of the model compound (BOD 1), energy-transfer cassette with self-reporting property (BOD 2), and the individual energy-transfer modules.

cleave the linker, liberating the fluorophore as a consequence. Fluorescence of the free $\mathbf{F L}$ is reinstated which is the reporter

Received: April 15, 2014

Published: May 21, 2014 
for the ${ }^{1} \mathrm{O}_{2}$ generation activity of the PS. A model compound with the same chemical architecture was also synthesized (Figure 1, BOD 1), which lack halogens on the core BODIPY, and as a consequence does not generate singlet oxygen efficiently due to prohibited intersystem crossing (ISC).

Absorption and fluorescence spectra of BOD 1 and BOD 2 and free donor and acceptor modules in acetonitrile are given in Figure 2. Characteristic absorption peaks of 2,6-ethyl-1,3,5,7-

\section{(a)}
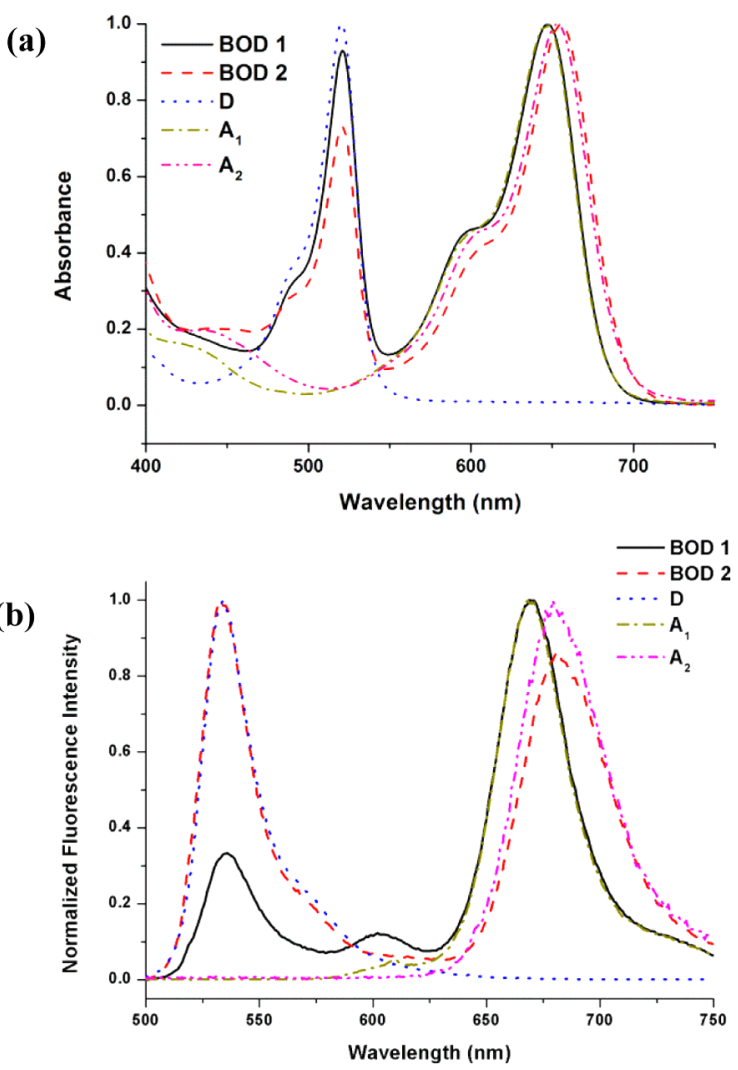

Figure 2. Normalized electronic absorption (a) and normalized emission (b) spectra of BOD 1, BOD 2, D, A1, and A2 in acetonitrile. Each spectrum is normalized with respect to maximum absorption or emission wavelength of each compound.

tetramethylBODIPYs at around $521 \mathrm{~nm}$ and distyryl-BODIPYs at $655 \mathrm{~nm}$ are measured. Compounds have two distinct emission peaks around $535 \mathrm{~nm}$ for donor emission, and 670 (BOD 1) or $681 \mathrm{~nm}$ (BOD 2) for acceptor emissions. Detailed photophysical data for the energy-transfer cassettes and the corresponding modules are given in Table 1 . As a consequence of EET, excitation with a $488 \mathrm{~nm}$ light, which is primarily absorbed by the donor, results in a decreased fluorescence from the donor module compared to the emission of the free donor BODIPY (Figure 3) with a decrease in quantum yield from 0.90 to 0.26 for BOD 1 and 0.34 for BOD 2 with a calculated energy transfer of $71 \%$ and $61 \%$ for the compounds, respectively. In BOD 1, emission at $670 \mathrm{~nm}$ is much more intense due to the lack of a competing intersystem crossing pathway in the acceptor module as it is in the case of halogenated acceptor module of BOD 2. Energy transfer is also calculated to be more pronounced in this model compound ( $71 \%$ in BOD 1 compared to $61 \%$ in BOD 2). This may be due to slightly larger overlap integral between the donor emission peak and the acceptor absorption peak in this molecule. The energy transfer processes in both compounds are further
Table 1. Photophysical Characterization of BOD 1 and BOD 2 and Modules A1, A2, and $\mathrm{D}^{a}$

$\begin{array}{lcccc} & \lambda_{\text {abs }}(\mathrm{nm}) & \varepsilon\left(\mathrm{M}^{-1} \mathrm{~cm}^{-1}\right) & \lambda_{\mathrm{F}}(\mathrm{nm}) & \phi_{\mathrm{F}}{ }^{b}\left(\lambda_{\text {exc }}(\mathrm{nm})\right) \\ \text { BOD 1 } & 521 & 61000 & 535 & 0.26 \\ & 647 & 65000 & 670 & 0.58 \\ \text { BOD 2 } & 521 & 59000 & 533 & 0.34 \\ & 655 & 97000 & 681 & 0.22 \\ \text { A1 } & 525 & 60000 & 670 & 0.53 \\ \text { A2 } & 654 & 95000 & 680 & 0.39 \\ \text { D } & 520 & 59000 & 534 & 0.90\end{array}$

${ }^{a}$ All values are measured in acetonitrile. ${ }^{b}$ For the emission band peaking at $521 \mathrm{~nm}$ Rhodamine 6G (water, $\phi_{\mathrm{F}}=0.95, n=1.333$ ) and for the emission band peaking at 670 and $680 \mathrm{~nm}$ Cresyl Violet ( $\left.\mathrm{MeOH}, \phi_{\mathrm{F}}=0.66, n=1.329\right)$ were used as reference compounds for quantum yield calculations. The refractive index of acetonitrile is taken as 1.346 .
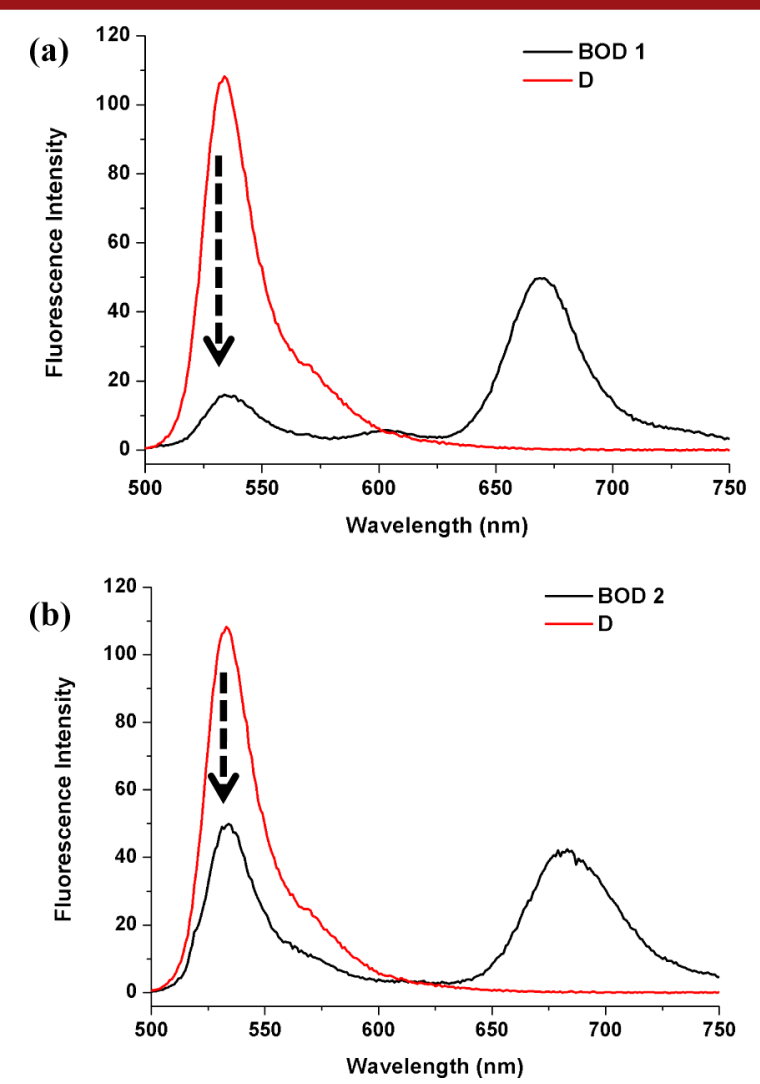

Figure 3. Fluorescence spectra of BOD 1 (a), BOD 2 (b), and equally absorbing donor module $\mathbf{D}$ in acetonitrile, excited at $488 \mathrm{~nm}$.

demonstrated by the excitation spectra. When the emission of the acceptor moiety is followed, excitation of the donor seems to contribute to this fluorescence whereas in the acceptor module itself, there is no corresponding excitability in the region of donor absorbance (Figures S1 and S2, Supporting Information).

Showing that the energy transfer is taking place between the modules in each compound (BOD 1 and BOD 2), the singlet oxygen dependent recovery of the donor emission is analyzed as an indication of PS activity. Efficient ${ }^{1} \mathrm{O}_{2}$ generation by the halogenated BODIPYs upon irradiation is already well studied. ${ }^{17-20}$ Here, each energy transfer systems are irradiated with a LED lamp $\left(660 \mathrm{~nm}, 2.5 \mathrm{~mW} / \mathrm{cm}^{2}\right.$ fluence rate). Primary red light absorbing species in the casettes are acceptor modules 
which are photosensitizer, or halogen-free fluorophore in BOD 2 and BOD 1, respectively. Aliquots were taken in $10 \mathrm{~min}$ intervals from the solutions of BOD 1 and BOD 2, and emission spectra were acquired by excitation at $520 \mathrm{~nm}$. The solutions were kept under irradiation with red light for the duration of the experiment. Singlet oxygen generated upon excitation of the photosensitizer is expected to react readily with the electron rich ( $Z$ )-1,2-bis(alkylthio)ethene linker and subsequently release donor fluorophore. This reaction can be monitored with the increased fluorescence of this module. As shown in Figures 4 and 5, control compound BOD 1 which
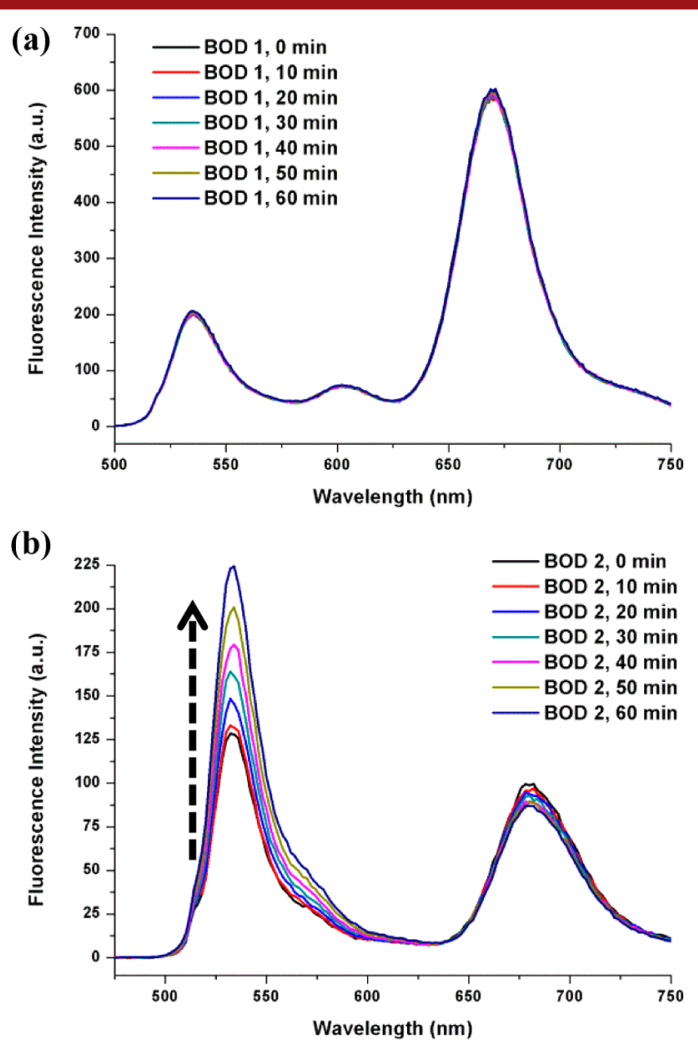

Figure 4. Change in fluorescence spectra of BOD 1 (a) and BOD 2 in dark and after irradiation with $660 \mathrm{~nm}$ light in acetonitrile (excited at $520 \mathrm{~nm}$ ).

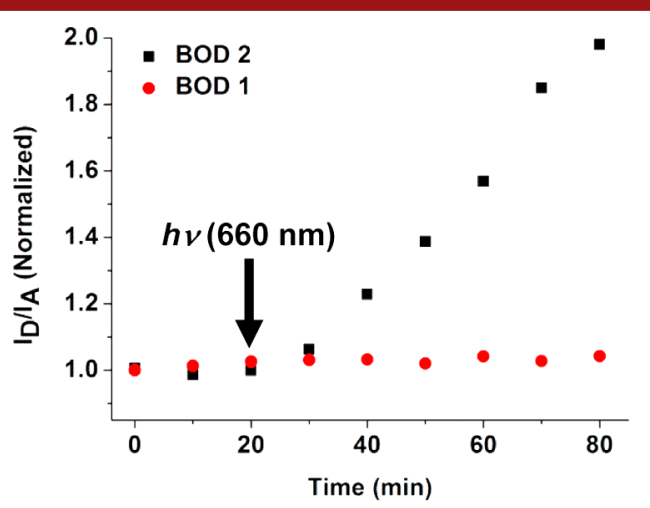

Figure 5. Normalized change in the fluorescence intensity of donor moiety in BOD 1 and BOD 2 (intensity at $532 \mathrm{~nm}$ ) compared to acceptor emission (intensity at $670 \mathrm{~nm}$ for BOD 1 and $680 \mathrm{~nm}$ for BOD 2) before $(0-20 \mathrm{~min})$ and after irradiation $(20-80 \mathrm{~min})$ with red light (excited at $520 \mathrm{~nm}$ ). lacks heavy atoms to facilitate intersystem crossing, display no significant change in emission spectra neither in dark, nor under irradiation at $660 \mathrm{~nm}$.

Just like BOD 1, BOD 2 shows no remarkable change in emission intensity when kept in dark (Figure 5). However, exposure to red light results in an almost 2-fold increase in donor emission, which is attributed to singlet oxygen dependent cleavage of the linker and subsequent disintegration of the EET system. Quantum yield of the donor moiety in BOD 2 restores back to free donor value within $80 \mathrm{~min}$, indicating complete cleavage of the ${ }^{1} \mathrm{O}_{2}$ susceptible bond within the given time scale. There is no significant change in acceptor emission in BOD 2 during the reaction. Hence, it is a useful reference point and comparison of the donor emission at 532 $\mathrm{nm}$ (which increase throughout the reaction) to this almost unchanged acceptor emission at $680 \mathrm{~nm}$ enables ratiometric activity sensing of the singlet oxygen (Figure 5).

Absorption spectra of the compounds throughout the singlet oxygen generation and reaction were also investigated, and no significant change is observed which can be interpreted as lack of any chemical change in the core BODIPYs during the reporting process, which would most likely yield distinct spectral signatures (Figures S3 and S4, Supporting Information).

In conclusion, we report a novel approach to monitor the activity of a photodynamic sensitizer, using singlet oxygen dependent release of a fluorescent reporter module of an energy transfer cassette, upon which fluorescence is restored. With a ratiometric comparison to the essentially unchanged acceptor emission, singlet oxygen generation ability of the photosensitizer can be assessed. The You group investigated ${ }^{16}$ a similarly designed system for its drug release potential. However, to the best of our knowledge, explicit reactionbased modulation of $\mathrm{EET}^{21}$ toward singlet oxygen-dependent ratiometric self-activity sensing of a photosensitizer has not been reported before. In any case, the rate of increase in the fluorescence of the reporter module is a direct measure of the rate of singlet oxygen generation under the conditions of the study. This system can be further developed for direct analysis of the activity of PDT agents in vivo. This will provide an unprecedented capability for in vivo assessment of photosensitizers for PDT in terms of singlet oxygen generation. This in turn, may lead to more advanced photosensitizer based therapeutics in the very near future.

\section{ASSOCIATED CONTENT}

\section{Supporting Information}

Experimental procedures, characterizations, and further spectroscopic data. This material is available free of charge via the Internet at http://pubs.acs.org.

\section{AUTHOR INFORMATION}

\section{Corresponding Author}

*E-mail: eua@fen.bilkent.edu.tr.

Notes

The authors declare no competing financial interest.

\section{ACKNOWLEDGMENTS}

S.E.-C. thanks TUBITAK for financial support. 


\section{REFERENCES}

(1) De Silva, A. P.; Gunaratne, H. Q. N.; Gunnlaugsson, T.; Huxley, A. J. M.; McCoy, C. P.; Rademacher, J. T.; Rice, T. H. Chem. Rev. 1997, 97, 1515-1566.

(2) Domaille, D. W.; Que, E. L.; Chang, C. J. Nature Chem. Bio. 2008, 4, 168-175.

(3) Lovell, J. F.; Liu, T. W. B.; Chen, J.; Zheng, G. Chem. Rev. 2010, 110, 2839-2857.

(4) McDonnell, S. O.; Hall, M. J.; Allen, L. T.; Byrne, A.; Gallagher, W. M.; O'Shea, D. F. J. Am. Chem. Soc. 2005, 127, 16360-16361.

(5) Yogo, T.; Urano, Y.; Mizushima, A.; Sunahara, H.; Inoue, T.; Hirose, K.; Iino, M.; Kikuchi, K.; Nagano, T. Proc. Natl. Acad. Sci. U.S.A. 2008, 105, 28-32.

(6) Erbas-Cakmak, S.; Bozdemir, O. A.; Cakmak, Y.; Akkaya, E. U. Chem. Sci. 2013, 4, 858-862.

(7) Ozlem, S.; Akkaya, E. U. J. Am. Chem. Soc. 2009, 131, 48-49.

(8) Chen, J.; Stefflova, K.; Niedre, M. J.; Wilson, B. C.; Chance, B.; Glickson, J. D.; Zheng, G. J. Am. Chem. Soc. 2004, 126, 11450-11451.

(9) Macdonald, I. J.; Dougherty, T. J. J. Porphyr. Phthalocya. 2001, 5, $105-129$.

(10) Cakmak, Y.; Kolemen, S.; Duman, S.; Dede, Y.; Dolen, Y.; Kilic, B.; Kostereli, Z.; Yildirim, L. T.; Dogan, A. L.; Guc, D.; Akkaya, E. U. Angew. Chem., Int. Ed. 2011, 50, 11937-11941.

(11) Rotaru, A.; Mokhir, A. Angew. Chem., Int. Ed. 2007, 46, 61806183.

(12) Voigt, N. V.; Torring, T.; Rotaru, A.; Jacobsen, M. F.; Raynsbaek, J. B.; Subrami, R.; Mamdouh, W.; Kjems, J.; Mokhir, A.; Besenbacher, F.; Gothelf, K. V. Nat. Nanotechnol. 2010, 5, 200-203.

(13) Nkepang, G.; Pogula, P. K.; Bio, M.; You, Y. Photochem. Photobiol. 2012, 88, 753-759.

(14) Erbas-Cakmak, S.; Akkaya, E. U. Angew. Chem., Int. Ed. 2013, 52, 11364-11368.

(15) Bio, M.; Rajaputra, P.; Nkepang, G.; Awuah, S. G.; Hossion, A. M. L.; You, Y. J. Med. Chem. 2013, 56, 3936-3942.

(16) Bio, M.; Nkepang, G.; Awuah, S. G.; You, Y. Chem. Commun. 2012, 48, 6517-6519.

(17) Erbas, S.; Gorgulu, A.; Kocakusakogullari, M.; Akkaya, E. U. Chem. Commun. 2009, 4956-4958.

(18) Yogo, T.; Urano, Y.; Ishitsuka, Y.; Maniwa, F.; Nagano, T. J. Am. Chem. Soc. 2005, 127, 12162-12163.

(19) Atilgan, S.; Ekmekci, Z.; Dogan, A. L.; Guc, D.; Akkaya, E. U. Chem. Commun. 2006, 4398-4400.

(20) Kamkaew, A.; Lim, S. H.; Lee, H. B.; Kiew, L. V.; Chung, L. Y.; Burgess, K. Chem. Soc. Rev. 2013, 42, 77-78.

(21) Guliyev, R.; Ozturk, S.; Kostereli, Z.; Akkaya, E. U. Angew. Chem., Int. Ed. 2011, 50, 9826-9831. 